\title{
Potential application of injectable chitosan hydrogel treated with siRNA in chronic rhinosinusitis therapy
}

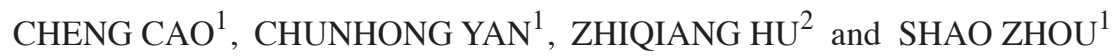 \\ ${ }^{1}$ Department of Otorhinolaryngology, Yinzhou Hospital Affiliated to the Medical School of Ningbo University; \\ ${ }^{2}$ Department of Otorhinolaryngology, No. 113 Hospital of PLA, Ningbo, Zhejiang 315000, P.R. China
}

Received November 9, 2014; Accepted July 28, 2015

DOI: $10.3892 / \mathrm{mmr} .2015 .4237$

\begin{abstract}
Chronic rhinosinusitis is a condition with severe clinical symptoms and limited therapeutic solutions. It has been reported that vascular endothelial growth factor (VEGF) can promote nasal epithelial cell growth and result in hyperplasia of the sinuses. Therefore, the downregulation of VEGF may inhibit the process of hyperplasia. In the present study, small interfering RNA (siRNA) targeting VEGF was used to silence the expression of VEGF, and injectable chitosan based hydrogel, which is suitable for sinus injection and exhibits long-term retention, was prepared as the siRNA carrier. Human bronchial epithelial cells were cultured directly on the hydrogel to observe the biological performance in vitro. Further in vivo effects were investigated by the injection of the hydrogel into the sinus cavity. Following the introduction of siRNA introducing, the expression of VEGF in the bronchial epithelial cells was significantly suppressed at mRNA and protein levels. The number of living cells on the gel was significantly decreased, thus resulting in the inhibition of proliferation. However, the cytoskeletal arrangement of the remaining cells were not affected substantially. The hydrogel was able to retain the siRNA for an extended duration, which enabled a sustained supply of siRNA. The in vivo sinus mucosa analysis revealed that the siRNA was able to collocate with cells and the mucosa thickness was substantially decreased. In conclusion, the results of the present study suggested that injectable chitosan based hydrogel, treated with siRNA targeting VEGF, may be used as a convenient therapeutic option for chronic rhinosinusitis.
\end{abstract}

Correspondence to: Dr Shao Zhou, Department of Otorhinolaryngology, Yinzhou Hospital Affiliated to the Medical School of Ningbo University, 251 East Baizhang Road, Ningbo, Zhejiang 315000, P.R. China

E-mail: shaozhou2014@163.com

Key words: rhinosinusitis, vascular endothelial growth factor, small interfering RNA, chitosan, injectable hydrogel

\section{Introduction}

Rhinosinustitis is one of the most prevalent upper airway problems (1). Despite the severe morbidity and impairment to the patient's quality of life, the therapeutic options are limited and recurrence is common (2). One of the most severe subtypes is chronic rhinosinusitis with hyperplastic sinonasal polyposis, the effects of which is marked as mucosal hyperplasia and tissue remodeling often exists. Often, the sinonasal polyposis requires surgical intervention, although recurrence is inevitable (3). Consequently, it remains critical to identify an internal medicine therapeutic solution. It is reported that vascular endothelial growth factor (VEGF) is key in the promotion of nasal epithelial cell growth and the inhibition of apoptosis, which results in hyperplastic sinonasal polyposis (4). Therefore, the present study hypothesized that downregulation of the local expression of VEGF may suspend the progress of chronic rhinosinustitis and prevent its substantial effects.

RNA interference technology represents a novel target gene silencing method. By internalizing of specific small interfering RNA (siRNA), it can bind to target mRNA, leading to cleavage of the mRNA and silencing of the target gene (5). This convenient and effective technique is extensively used in various areas, including cancer therapy (6), regenerative medicine (7), anti-viral therapy (8) and anti-inflammatory treatment (9), therefore, siRNA targetting VEGF may be a therapeutic molecule in chronic rhinosinustitis. Chitosan is a natural cationic polymer with positive charge, and has been used as an important siRNA non-viral vector due to its advantages, including lower cost and notable cytocompatibility (10). A vital property of chitosan is mucosa adhesion, which suggests that chitosan may be a suitable drug delivery carrier in mucosa lesions (11). In addition, chitosan-based thermosensitive hydrogel, also known as injectable hydrogel, which is fluid at low temperature and solid at body temperature, can retain siRNA for a prolonged duration $(12,13)$ and may be suitable for sinus injection.

In the present study, siRNA targeting VEGF (siVEGF) was delivered into nasal epithelial cells in injectable chitosan hydrogel to obtain localized sustained VEGF silencing. Subsequent analysis of cell proliferation and the cytoskeleton were performed to evaluate the in vitro biofunction, and mucosal thickness was determined to assess the degree 
of inflammation following injection of the hydrogel into the maxillary sinus of a chronic rhinosinusitis animal model.

\section{Materials and methods}

Materials. Chitosan (Mw, 100 300 kDa; DD=93.37\%), sodium $\beta$-glycerophosphate ( $\beta$-GP), 3-(4,5-dimethylthiazol-2yl)-2, 5-diphenyltetrazolium bromide (MTT) and dimethyl sulfoxide (DMSO) were purchased from MP Biomedicals, Santa Ana, CA, USA. Fluorescein isothiocyanate (FITC)-conjugated phalloidin, DAPI and the live/dead cell staining kit were purchased from Nanjing KeyGen Biotech. Co., Ltd. (Nanjing China). Human VEGF siRNA (sense 5'-GGAGUACCCUGAUGAGAUCdTdT-3'; antisense 5'-GAUCUCAUCAGGGUACUCCdTdT-3') and siRNA negative control (sense 5'-UUCUCCGAACGUGUCACGUTT-3'; antisense 5'-ACGUGACACGUUCGGAGAATT-3') were purchased from Shanghai Genepharma, Co., Ltd. (Shanghai, China). The RiboGreen kit and TRIzol reagent was purchased from Invitrogen Life Technologies (Carlsbad, CA, USA). The PrimeScript ${ }^{\mathrm{TM}}$ RT reagent kit and SYBR Premix $\mathrm{Ex}^{\mathrm{TM}}$ Taq II were purchased from Takara Bio, Inc. (Otsu, Japan). The Human VEGF Enzyme-Linked Immunosorbent Assay (ELISA) kit was purchased from antibodies-online $\mathrm{GmbH}$ (Aachen, Germany).

Fabrication of the hydrogel. The injectable chitosan hydrogel was fabricated, according to a previous report (12). Briefly, chitosan was dissolved in $1 \%$ acetic acid at a concentration of $2 \% \mathrm{w} / \mathrm{v}$. The $\beta$-GP was prepared in distilled water at a $1 \mathrm{mg} / \mathrm{ml}$ concentration. To fabricate the hydrogel, $1.8 \mathrm{ml}$ chitosan solution was stirred constantly at $4^{\circ} \mathrm{C}$, and $0.2 \mathrm{ml} \beta$-GP solution was added in a drop-wise manner. The hydrogel spontaneously became solid during incubation at $37^{\circ} \mathrm{C}$ for $8 \mathrm{~min}$ (Fig. 1). To incorporate the siRNA, pre-calculated siRNA $(5 \mu \mathrm{g})$ was added into the $200 \mu \mathrm{l}$ liquid solution at $4^{\circ} \mathrm{C}$ prior to the gel formation.

Characterization of the gel. The gels, which had been either loaded with siRNA or were siRNA-free, were freeze-dried and sputter coated with platinum. The gels were then observed using a scanning electron microscope (SEM; S-4800; Hitachi, Ltd., Toyko, Japan). In addition, Cy3-labeled siRNA (Cy3-siRNA) was used during gel formulation and was observed using a fluorescence microscope (Olympus Corporation, Toyko, Japan).

The in vitro siRNA release profile was determined separately under neutral $(\mathrm{pH}$ 7.4) and acidic $(\mathrm{pH} 5.5)$ phosphate-buffered saline (PBS). Specifically, the hydrogel was soaked in the respective PBS and incubated under cell culture conditions for 2 weeks. At predetermined time-points, the elution was collected and replaced with fresh buffer. The released siRNA in the supernatant was quantified using the RiboGreen kit, according to the manufacturer's instructions. Briefly, $100 \mu \mathrm{l}$ sample was thoroughly mixed with $100 \mu \mathrm{l}$ RiboGreen in the dark. Each sample was duplicated three times and the fluorescence intensity was measured using a fluorescence plate reader (TECAN Infinite M200; Tecan, Männedorf, Switzerland) at the excitation and emission wavelengths of 546 and $596 \mathrm{~nm}$, respectively. Fresh PBS was used as blank control and the relative fluorescent units (RFUs) were determined by subtracting the blank fluorescence intensity. The siRNA was calculated using a calibration curve and the percentage of cumulative release was calculated using Microsoft Excel 2013 (Microsoft, Redmond, VA, USA).

Cell culture and seeding. The 16HBE bronchial epithelial cell line was cultured in bronchial epithelial basal medium (Biosource, Camarillo, TX, USA). The cell seeding method used was in accordance to that of a previous report with modification (14). The culture conditions were $37^{\circ} \mathrm{C}$ in $5 \% \mathrm{CO}_{2}$ and $100 \%$ humidity. When the confluence of the cells was at $~ 80 \%$, the cells were trypsinized $(0.25 \%$ trypsin and $0.01 \%$ EDTA; GE Healthcare Life Sciences, Logan, UT, USA and the density adjusted to $2 \times 10^{6}$ cells $/ \mathrm{ml}$. Subsequently, $200 \mu \mathrm{l}$ was added to the gel and placed in a 24-well tissue culture plate (TCP), $1 \mu \mathrm{l}$ medium was supplemented following $4 \mathrm{~h}$ attachment.

siRNA uptake and gene knockdown. To determine the uptake of siRNA from the gel, the Cy3-labeled human VEGF siRNA (Cy3-siRNA) was loaded and incubated with the cells for $24 \mathrm{~h}$. The cells were then trypsinized from the gel and re-seeded onto the TCP. The cells were allowed to attach for another $24 \mathrm{~h}$ and observed using a fluorescence microscope.

At pre-determined time-points, the cells were lysed with TRIzol reagent, and the total RNA was extracted using chloroform and precipitated in isopropanol (Tianjin Kemiou Chemical Reagent Co., Ltd., Tianjin, China). Following quantification by measurement of optical density (OD) at the wavelength of $260 \mathrm{~nm}$ using an Epoch plate reader (BioTek Instruments, Winooski, VT, USA), $1 \mu \mathrm{g}$ total RNA was used reverse transcribed into cDNA using the PrimeScript ${ }^{\mathrm{TM}}$ RT reagent kit. The mRNA expression of target VEGF was analyzed using quantitative polymerase chain reaction (qPCR) with an SYBR Premix ExTM Taq II kit (Takara Bio, Inc.). The primers used were as follows: Human VEGF sense 5'-GCACCCATGGCAGAAGGAGG-3' and antisense 5'-CCTTGGTGAGGTTTGATCCGCATA-3'; human GAPDH sense 5'-CGGATTTGGTCGTATTGGGC-3' and antisense 5'-GTCATACCAGGAAATGAGCTTG-3'. A $10 \mu \mathrm{l}$ system was used, containing $5 \mu \mathrm{l} \mathrm{SYBR}$ Premix, $1 \mu \mathrm{l}$ forward primer, $1 \mu \mathrm{l}$ reverse primer and $3 \mu \mathrm{l}$ cDNA. The thermocycling was repeated between $95^{\circ} \mathrm{C}(15 \mathrm{sec})$ and $60^{\circ} \mathrm{C}(30 \mathrm{sec})$ for 40 cycles. The expression was determined by the threshold cycle $(\mathrm{Ct})$ value of each well. The protein level of VEGF in cell supernatants were determined using ELISA kits, according to the manufacturer's instructions. Briefly, the samples were incubated in human VEGF antibody-coated wells and colorized using horesradish peroxidase catalysis. The absorbance was read at $450 \mathrm{~nm}$ and the concentration was calculated based on the calibration curve. Data are expressed as per $\mu \mathrm{g}$ total protein, which was measured using a bicinchoninic acid assay kit (Sigma-Aldrich).

Proliferation analysis and staining for determination of cell death. The proliferation of the cells grown on the different substrate gels were analyzed continuously using an MTT assay. Briefly, the cells were incubated with MTT reaction solution $(5 \mathrm{mg} / \mathrm{ml} \mathrm{MTT}$ : medium, $1: 4)$ for $4 \mathrm{~h}$ at $37^{\circ} \mathrm{C}$. Subsequently, the supernatants were gently removed and 

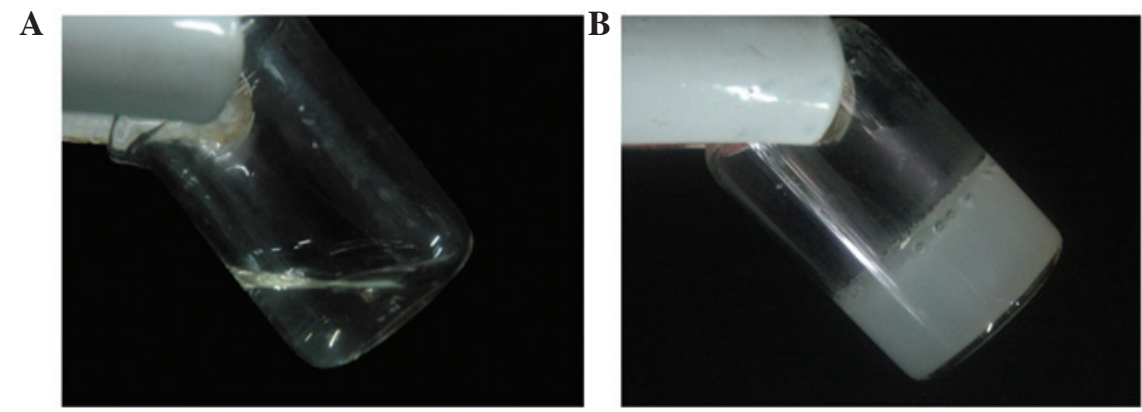

Figure 1. Formulation of the injectable chitosan based hydrogel. (A) Solution state at $4^{\circ} \mathrm{C}$. (B) Hydrogel state at $37^{\circ} \mathrm{C}$.

the precipitation was completely dissolved in DMSO. The OD value was measured using a spectrometer at $490 \mathrm{~nm}$. Following 3 days of culture, the medium was removed and the cells were rinsed with PBS. The cells were then stained using the Live/Dead cells staining kit and observed using a fluorescence microscope.

Actin cytoskeletal staining. Following 3 days of culture on the hydrogel, the cells were fixed in $4 \%$ paraformaldehyde (Beijing Leagene Biotech Co., Ltd., Beijing, China) and then permeabilized with $0.1 \%$ Triton-X100 (Beijing Leagene Biotech Co., Ltd.). To reveal the actin cytoskeleton, the cells were stained with FITC-conjugated phalloidin solution $(50 \mathrm{mg} / \mathrm{ml}$ in PBS) for $20 \mathrm{~min}$ at room temperature. The unbound dye was washed thoroughly with PBS and the samples were observed under a fluorescence microscope.

Animal model establishment and hydrogel injection. The animal procedures used in the present study were approved by the research ethics committee of the Medical School of Ningbo University (Ningbo, China). A total of 20 male Sprague-Dawley rats (6-8 weeks old) were purchased from the School of Medicine, Ningbo University (Ningbo, China) and each group contained five rats. The rats were housed separately at $20-25^{\circ} \mathrm{C}$ under a 12 -h light/dark cycle and fed a normal diet. The chronic rhinosinusitis animal model was established according to a previous report (15). Following establishment of the model, the hydrogel containing the different siRNA was injected $(10 \mu \mathrm{l})$, immediately following formulation, into the bilateral maxillary sinus. The animal was sacrificed 2 weeks post-injection via intra-abdominal overdose of pentobarbital sodium solution, and the sinus was isolated carefully for further analysis.

Prolonged mucosal siRNA delivery and measurement of mucosal thickness. The Cy3-labeled siRNA had been incorporated into the gel prior to injection, with the same quantity of siRNA in the chitosan/siRNA nanoparticles (NPs) or free siRNA injected. Following animal sacrifice, the sinus mucosa was stripped off and the thickness of the sinus mucosa was measured using graduated probe at five randomly-selected points. The sample was then fixed in $4 \%$ paraformaldehyde and cryosectioned, followed by DAPI staining. The quantitative siRNA concentrations were determined according to previous report (16). Briefly, the mucosa was homogenized and mixed with lysis buffer $(0.1 \%$ sodium dodecyl sulfate in
PBS; Beijing Leagene Biotech Co., Ltd.) and incubated at $65^{\circ} \mathrm{C}$ for $10 \mathrm{~min}$, followed by the addition of methanol and incubation for a further $10 \mathrm{~min}$. The sample was then centrifuged at $12,000 \mathrm{x} \mathrm{g}$ for $5 \mathrm{~min}$ at $4^{\circ} \mathrm{C}$, and $200 \mu \mathrm{l}$ of the supernatant was transferred to a black 96-well plate (Corning Incorporated, Corning, NY, USA). The fluorescence intensity of the sample was measured using an Epoch plate reader ( $\lambda \mathrm{ex}, 554 \mathrm{~nm} ; \lambda \mathrm{em}$, $568 \mathrm{~nm}$ ). The concentration of Cy3-siRNA in each sample was calculated from a standard curve.

Statistical analysis. All experiments were performed triplicates and are presented as the mean \pm standard deviation. One-way analysis of variance and the Student-Newman-Keuls test were performed to analyze the data using SPSS 22.0 (IBM SPSS, Armonk, NY, USA). P<0.05 was considered to indicate a statistically significant difference.

\section{Results}

Characterizations of the hydrogel. The hydrogel was visualized using SEM and a fluorescence microscope separately (Fig. 2A). The gel exhibited a porous structure with numerous protuberant particles (Fig. 2Aa). Following the loading of siRNA, the porous structure exhibited minimal change, however the particles became more visible (Fig. 2Ab). Compared with the siRNA-free gel (Fig. 2Ac), loading of Cy3-siRNA produced a self-organized red fluorescence signal (Fig. 2Ad). The siRNA release profile from the gel was then determined. The release profile under different $\mathrm{pH}$ values was determined within 2 weeks to simulate the natural body fluid condition. The siRNA release occurred gradually under neutral conditions, without apparent initial burst release, and siRNA was maintained for a prolonged duration (Fig. 2B). When the gel was exposed to the acidic condition, the release rate was markedly increased and a burst release was observed within the first day (Fig. 2B).

Uptake of siRNA and target gene silencing. The cells were detached from the Cy3-siRNA-loaded hydrogel and reattached onto a tissue culture plate to observe the uptake behavior. As indicated, almost all the red fluorescence signals were observed within the cells (Fig. 3A). The in vitro capability of the target silencing effect within the $16 \mathrm{HBE}$ cells was first analyzed (Fig. 3B). The mRNA level of VEGF was suppressed by $\sim 20 \%$ at day 3 , and the silencing effect was higher on days 5 and 7, by $\sim 40 \%$ (Fig. 3B). In agreement 
A

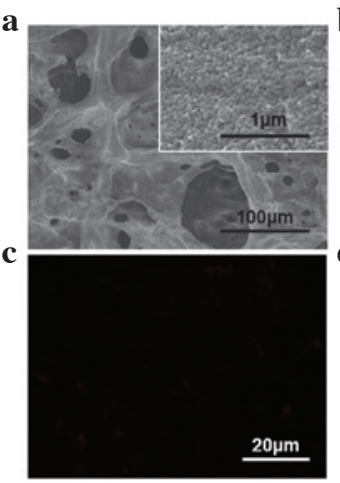

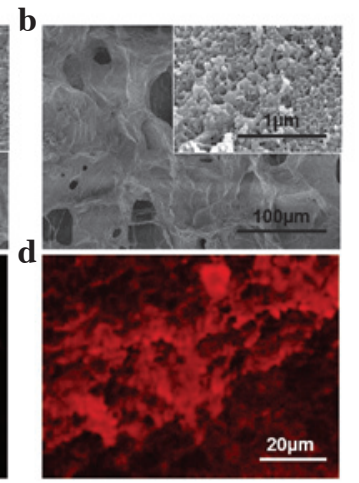

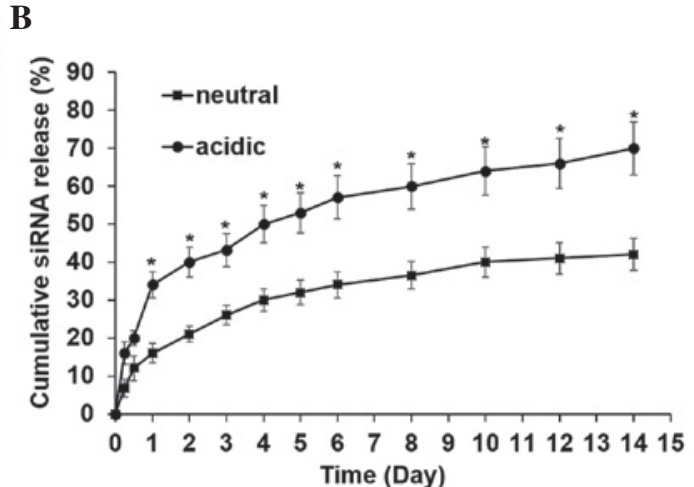

Figure 2. Surface observation of the (Aa) siRNA-free gel and the (Ab) gel loaded with siRNA using scanning electron microscopy and (Ac and d) fluorescence microscope, respectively. (B) Cumulative siRNA release profile under neutral and acidic conditions, determined using the RiboGreen assay. Data are expressed as the mean \pm standard deviation. ${ }^{*} \mathrm{P}<0.05$, compared with neutral. siRNA, small interfering RNA.

\section{A}
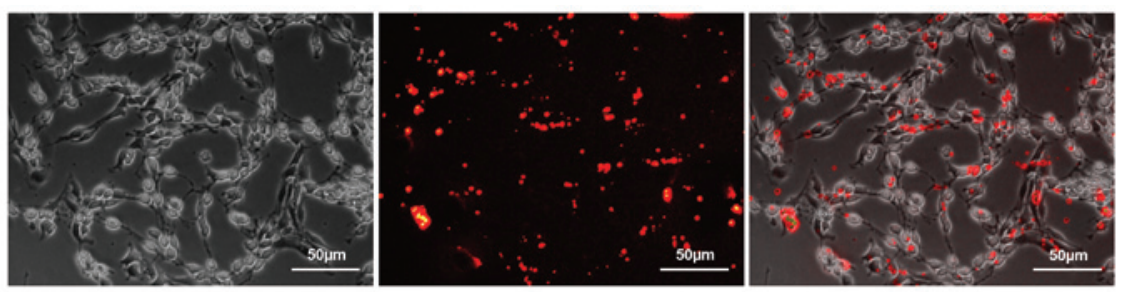

B

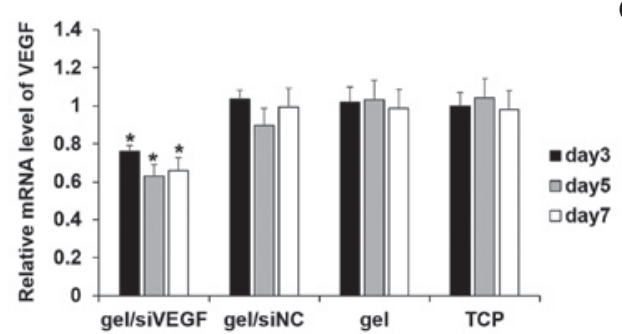

$\mathrm{C}$

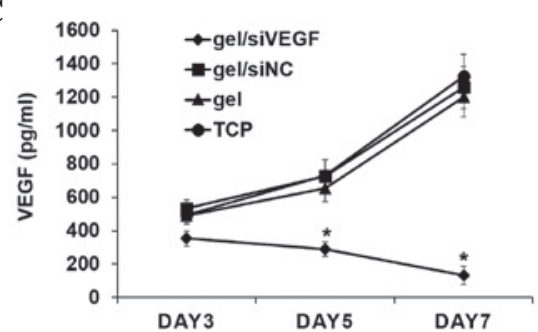

Figure 3. (A) Visualization of the uptake of siRNA using fluorescence microscopy. (B) mRNA expression of VEGF, determined using quantitative polymerase chain reaction analysis. (C) Associated VEGF protein level, determined by enzyme-linked immunosorbent assays. "P $<0.05$, compared with the other three control groups. Data are expressed as the mean \pm standard deviation. siRNA, small interfering RNA; siNC, siRNA negative control; VEGF, vascular endothelial growth factor; TCP, tissue culture plate.
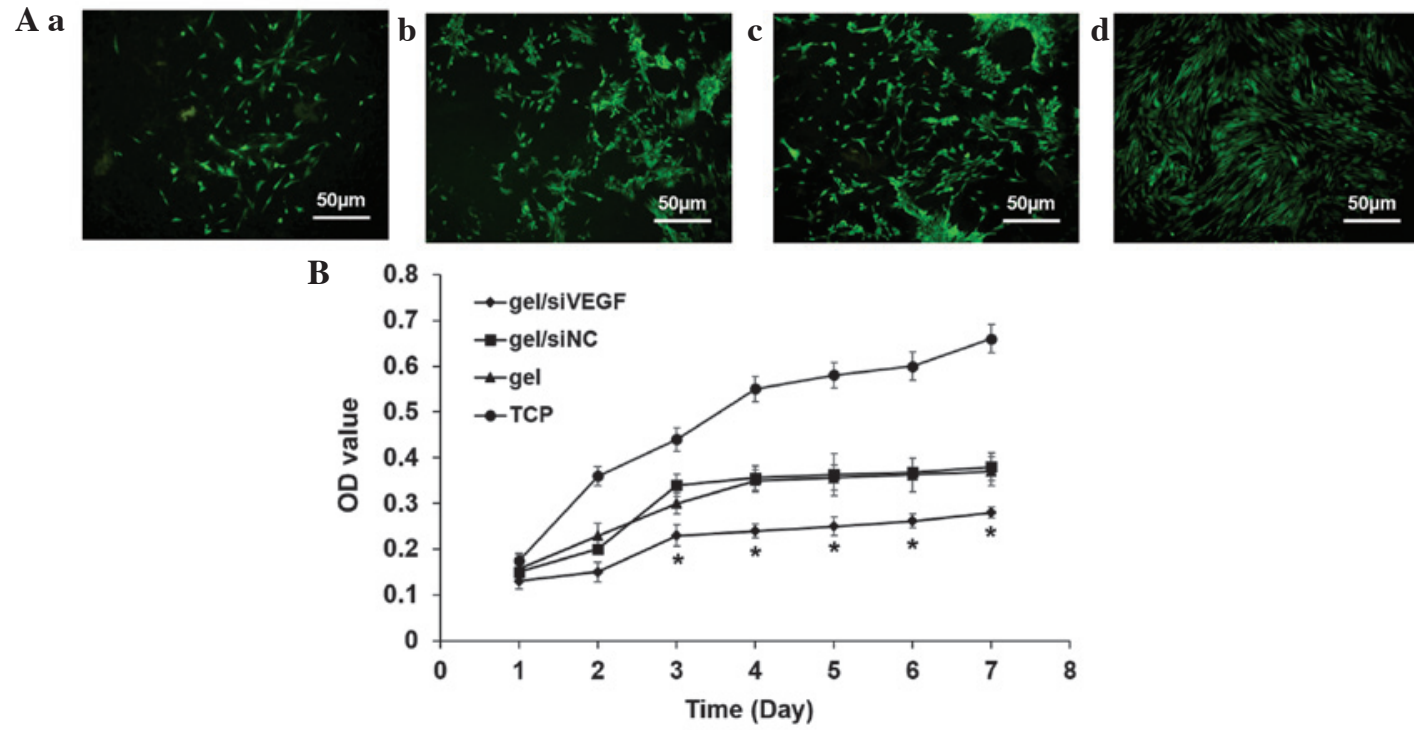

Figure 4. siRNA decreases proliferation and viability of 16HBE cells. (A) Staining with a Live/Dead cell staining kit to determine the number of living cells in the (a) gel/siVEGF; (b) gel/siNC; (c), gel; and (d) TCP groups. (B) Proliferation of epithelial cells on different hydrogels. "P<0.05, compared with the other three contros. Data are expressed as the mean \pm standard deviation. siRNA, small interfering RNA; siNC, siRNA negative control; VEGF, vascular endothelial growth factor; TCP, tissue culture plate; OD, optical density. 


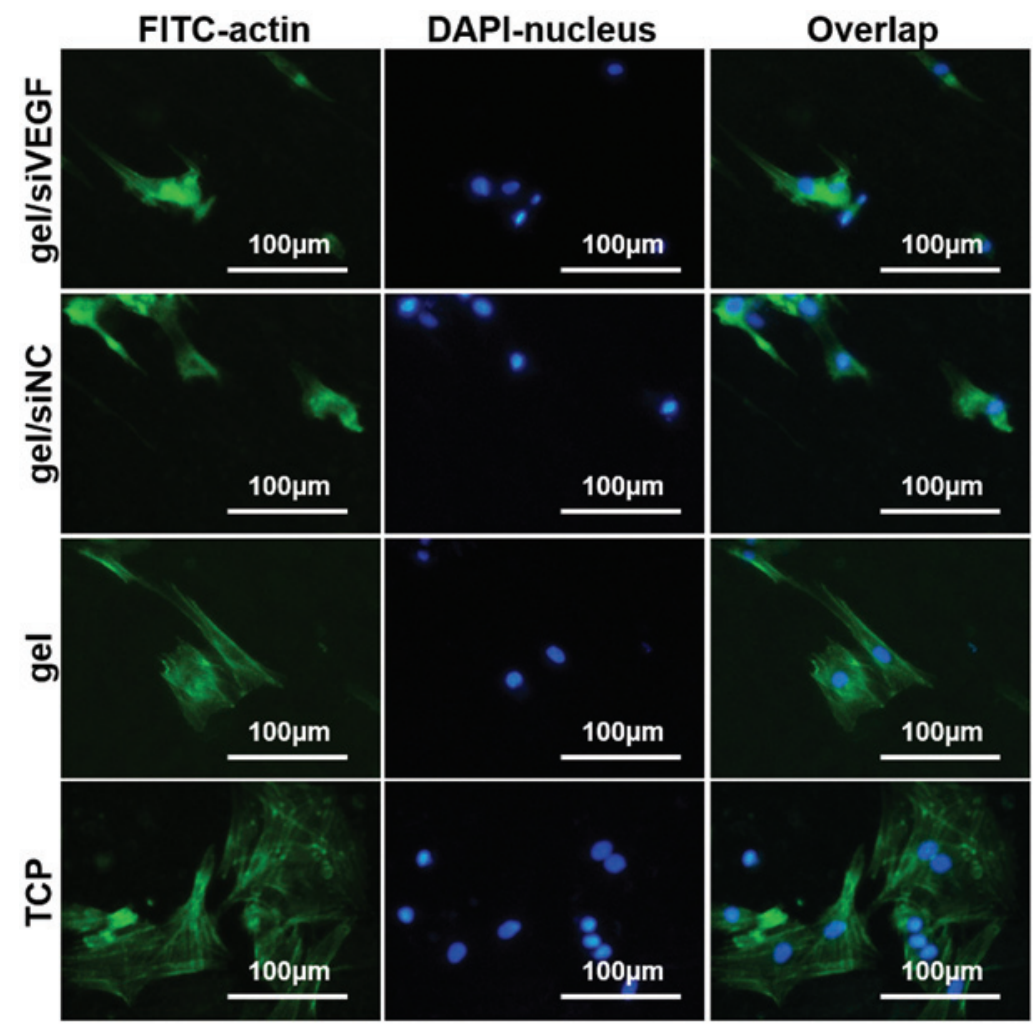

Figure 5. Actin cytoskeleton staining of the $16 \mathrm{HBE}$ cells grown on different hydrogels. Data are expressed as the mean \pm standard deviation. siRNA, small interfering RNA; siNC, siRNA negative control; VEGF, vascular endothelial growth factor; TCP, tissue culture plate; FITC, fluorescein isothiocyanate.

A

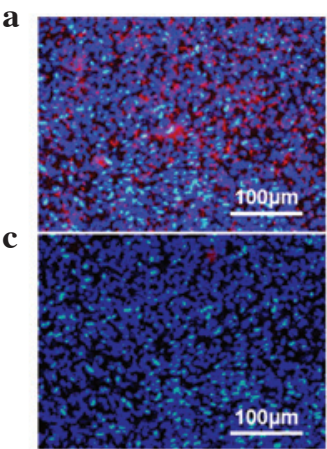

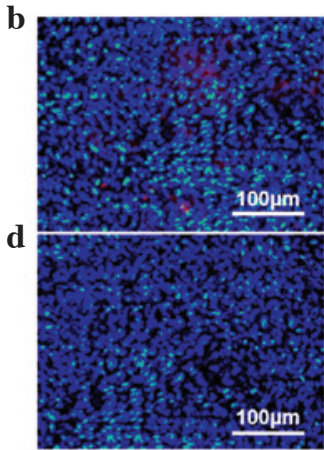
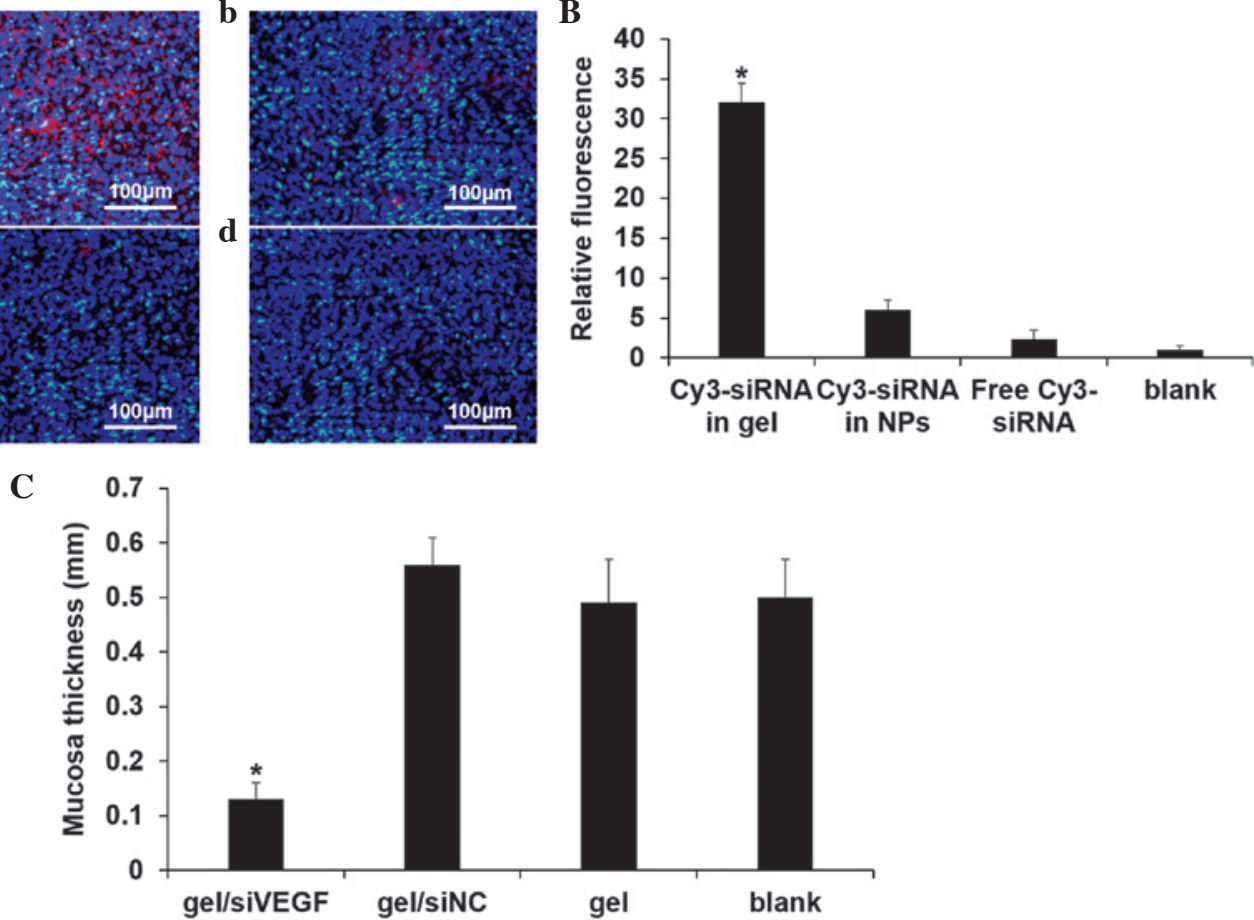

Figure 6. siRNA provides prolonged co-localization. (A and B) Prolonged siRNA co-localization within the sinus mucosa. (a) Cy3-siRNA in gel; (b) Cy3-siRNA in NPs; (c) free Cy3-siRNA; and (d) blank. (C) Measurement of sinus mucosa thickness. "P<0.05, compared with the other three control groups. Data are expressed as the mean \pm standard deviation. siRNA, small interfering RNA; siNC, siRNA negative control; NPs, nanoparticles; VEGF, vascular endothelial growth factor; TCP, tissue culture plate.

with the results of the qPCR analysis, the protein level of VEGF in supernatant was markedly downregulated on the gel loaded with siVEGF (Fig. 3C).
Inhibition of cell proliferation. Following 3 days culture, the number of living cells deceased on all the gels, compared with the TCP and was significantly reduced on the gel loaded with 
siVEGF (Fig. 4A). The cell vitality was monitored continuously for 1 week to determine the proliferation. Regardless of the type of gel loading, the cell proliferation was downregulated, compared with the TCP group (Fig. 4B). On the siVEGF-loaded gel, cell vitality was suppressed significantly, compared with all the other groups (Fig. 4B).

Actin cytoskeleton arrangement. To examine the spread of the cells grown on the gels, the actin cytoskeleton was stained. In general, the cells grown on gels were shrunken and exhibited a spindle-like shape, whereas the cells grown on TCP were spread widely with high levels of cell-cell contact (Fig. 5). The changes were particularly marked on the gel loaded with siVEGF (Fig. 5).

Sustained delivery of siRNA in vivo and mucosa thickness measurement. The sinus mucosa was isolated to analyze the gel function in vivo. Following 2 weeks of intervention, only the siRNA in the gel was able to co-localize with the mucosal cells, exhibiting $>30$-fold higher fluorescence intensity (Fig. 6Aa). Direct injection of the chitosan/siRNA NPs were able to co-localize to a certain degree, however, the long term preserving ability was deficient (Fig. 6Ab). Quantification is shown in Fig.6 B. The sinus mucosa thickness was determined to reflect the inflammation inhibition capability. The thickness was reduced significantly following the sustained silencing of VEGF, suggesting that the incrassated mucosa caused by rhinosinusitis was withdrawn (Fig. 6C).

\section{Discussion}

Chronic rhinosinusitis is a debilitating condition in humans and the enlarged nasal polyposis often requires a surgical procedure, which is often relapses and can be problematic (17). Therefore, the identification of novel internal medical treatments for chronic rhinosinusitis is imperative. In the present study, downregulation of the expression of VEGF, one of the key molecules during the formation of incrassated mucosa, was performed to determine its effect on rhinosinusitis inhibition. VEGF has a close association with the development of rhinosinusitis. VEGF is abundantly expressed by nasal epithelial cells and promotes cell hyperplasia in polyposis (4), and other members of the VEGF gene family of ligands and receptors are also highly expressed (18). In addition, VEGF can increase vascular permeability and in present in the nasal secretions produced in rhinosinusitis (19). Therefore, utilization of siVEGF may inhibit epithelial cell function and thus inhibit the process of rhinosinusitis. It was also observed in the present study that epithelial cells proliferation is significantly suppressed following downregulation if the expression of VEGF and resulted in a decreased autocrine effect.

In order to obtain sustained localized siRNA delivery and silencing, the injectable chitosan hydrogel was selected for use in the present study. As a thermosensitive hydrogel, which transfers to a solid state under physiological conditions, it is convenient for localized injection and is retained in situ without surgical procedure. Notable, the injectable chitosan hydrogel has been extensively investigated in several areas, including periodontal regeneration (13), tumor therapy (12) and tissue repair (20). The sinuses are located within the maxillofacial bones, which are difficult to access in medicine intervention. Consequently, injectable hydrogel may be preferable for localized sinuses drug delivery (20). Another beneficial property of chitosan hydrogel is the long-term preservation of the drugs loaded onto it, which is ideal for sustained local drug delivery. In the present study, it was observed that siRNA was released gradually in neutral conditions and at a higher rate in acidic conditions, which can be explained by the fast dissolution of chitosan in acidic conditions. The local $\mathrm{pH}$ of tissue is considered to decrease in the presence of inflammation exists (21). Therefore, it may trigger the burst release of siRNA from the hydrogel within the lesion area at the initial stage, which is essential to provide sufficient siRNA penetration into the mucosa. In the present study, due to the ability of the hydrogel to provide sustained delivery, the siRNA collocated with the mucosa cells remained detectable after 2 weeks, which was not observed following use of the NP system or with free siRNA. The chitosan molecule is mucoadhesive and as the present study observed that the siRNA collocated with the mucosa cells, this suggested that it may be used as a potential mucosa target drug delivery system $(11,22)$. The mucosal thickness can be used to reflect the level of inflammation (23) and the present study demonstrated that it was markedly decreased following siVEGF introducing. In conclusion, the present study described a convenient chronic rhinosinusitis therapeutic strategy using the technique of injecting chitosan hydrogel containing siRNA. This drug delivery system offers considerable advantages for the specific sinus structure and may also be used to deliver other bioactive factors for more beneficial effects.

\section{Acknowledgements}

The present study was supported technically by the State Key Laboratory of Stomatology, the Fourth Military Medical University.

\section{References}

1. Obaseki D, Potts J, Joos G, Baelum J, Haahtela T, Ahlström M, Matricardi P, Kramer U, Gjomarkaj M, Fokkens W, et al: The relation of airway obstruction to asthma, chronic rhinosinusitis and age: Results from a population survey of adults. Allergy 69: 1205-1214, 2014.

2. Van Zele T, Holtappels G, Gevaert P and Bachert C: Differences in initial immunoprofiles between recurrent and nonrecurrent chronic rhinosinusitis with nasal polyps. Am J Rhinol Allergy 28: 192-198, 2014.

3. Saedi B, Sadeghi M, Akhavan-Khaleghi N and Seifmanesh H: Impact of endoscopic sinus surgery on the quality of life of patients with nasal polyposis. B-ENT 10: 59-65, 2014.

4. Lee HS, Myers A and Kim J: Vascular endothelial growth factor drives autocrine epithelial cell proliferation and survival in chronic rhinosinusitis with nasal polyposis. Am J Respir Crit Care Med 180: 1056-1067, 2009.

5. Felipe AV, Oliveira J, Chang PY, Moraes AA, da Silva TD, Tucci-Viegas VM and Forones NM: RNA interference: A promising therapy for gastric cancer. Asian Pac J Cancer Prev 15: 5509-5515, 2014.

6. Sahin B, Fife J, Parmar MB, Valencia-Serna J, Gul-Uludağ H, Jiang X, Weinfeld M, Lavasanifar A and Uludağ H: siRNA therapy in cutaneous T-cell lymphoma cells using polymeric carriers. Biomaterials 35: 9382-9394, 2014.

7. Gu X, Ding F and Williams DF: Neural tissue engineering options for peripheral nerve regeneration. Biomaterials 35: 6143-6156, 2014. 
8. Fu GF, Pan JC, Lin N, Hu HY, Tang WM, Xu JS, Wang XL, Xu XQ, Qiu T, Liu XY, et al: siRNA against KIR3DL1 as a potential gene therapeutic agent in controlling HIV-1 infection. Viral Immunol 27: 207-213, 2014.

9. Howard KA, Paludan SR, Behlke MA, Besenbacher F, Deleuran B and Kjems J: Chitosan/siRNA nanoparticle-mediated TNF-alpha knockdown in peritoneal macrophages for anti-inflammatory treatment in a murine arthritis model. Mol Ther 17: 162-168, 2009.

10. Rudzinski WE and Aminabhavi TM: Chitosan as a carrier for targeted delivery of small interfering RNA. Int J Pharm 399: $1-11,2010$

11. Gonçalves IC, Henriques PC, Seabra CL and Martins MC: The potential utility of chitosan micro/nanoparticles in the treatment of gastric infection. Expert Rev Anti Infect Ther 12: 981-992, 2014.

12. Han HD, Mora EM, Roh JW, Nishimura M, Lee SJ, Stone RL, Bar-Eli M, Lopez-Berestein G and Sood AK: Chitosan hydrogel for localized gene silencing. Cancer Biol Ther 11: 839-845, 2011

13. Ma Z, Yang C, Song W, Wang Q, Kjems J and Gao S: Chitosan hydrogel as siRNA vector for prolonged gene silencing. J Nanobiotechnology 12: 23, 2014.

14. Andersen M $\varnothing$, Le DQS, Chen M, Nygaard JV, Moustapha K, Bünger $C$ and Kjems J: Spatially controlled delivery of siRNAs to stem cells in implants generated by multi-component additive manufacturing. Adv Funct Mater 23: 5599-5607, 2013.

15. Ahn SK, Jeon SY, Khalmuratov R, Kim DJ, Kim JP, Park JJ and Hur DG: Rat model of staphylococcal enterotoxin B-induced rhinosinusitis. Clin Exp Otorhinolaryngol 1: 24-28, 2008.
16. Li SD, Chen YC, Hackett MJ and Huang L: Tumor-targeted delivery of siRNA by self-assembled nanoparticles. Mol Ther 16: 163-169, 2008.

17. Rimmer J, Fokkens W, Chong LY and Hopkins C: Surgical versus medical interventions for chronic rhinosinusitis with nasal polyps. Cochrane Database Syst Rev 12: CD006991, 2014.

18. Lee HS and Kim J: Constitutive expression of vascular endothelial cell growth factor (VEGF) gene family ligand and receptors on human upper and lower airway epithelial cells. Int Forum Allergy Rhinol 4: 8-14, 2014.

19. Matsune S, Ohori J, Yoshifuku K and Kurono Y: Effect of vascular endothelial growth factor on nasal vascular permeability. Laryngoscope 120: 844-848, 2010.

20. Burdick JA: Injectable gels for tissue/organ repair. Biomed Mater 7: 020201, 2012.

21. Punnia-Moorthy A: Evaluation of $\mathrm{pH}$ changes in inflammation of the subcutaneous air pouch lining in the rat, induced by carrageenan, dextran and staphylococcus aureus. J Oral Pathol 16: 36-44, 1987.

22. Fernandes M, Gonçalves IC, Nardecchia S, Amaral IF, Barbosa MA and Martins MC: Modulation of stability and mucoadhesive properties of chitosan microspheres for therapeutic gastric application. Int J Pharm 454: 116-124, 2013.

23. Kinis V, Ozbay M, Akdag M, Alabalık U, Gul A, Yılmaz B, Ozkan H and Topcu I: Effects of caffeic acid phenethyl ester on wound healing of nasal mucosa in the rat: An experimental study. Am J Otolaryngol 35: 482-486, 2014. 